\title{
The TAROT observatory data management
}

\author{
M. Bringer ${ }^{1}$, M. Boër ${ }^{1}$, C. Peignot ${ }^{1}$, G. Fontan ${ }^{2}$, and C. Merce $^{2}$ \\ 1 Centre d'Études Spatiales des Rayonnements, CNRS, 9 Av. du Colonel Roche, 31028 Toulouse Cedex 04, France \\ ${ }^{2}$ Laboratoire d'Analyses et d'Architectures des Systèmes, 7 Av. du Colonel Roche, 31077 Toulouse Cedex 04, France
}

Received December 18, 1998; accepted March 12, 1999

\begin{abstract}
TAROT (Télescope à Action Rapide pour les Objets Transitoires, Rapid Action Telescope for Transient Objects) is an autonomous ground based observatory (Calern, France) whose primary goal is the rapid detection of variable objects, peculiarly optical counterparts of Gamma Ray Burst (GRB) sources. We present the observatory data management architecture which is composed of 3 main modules: The MAJORDOME module whose aim is to optimally schedule the observation requests sent to the telescope through socket connections, e-mail or even a web interface, The CONTROL module which monitors the hardware, and a data processing software TAITAR which detects, deblends, measures, classifies sources and detects variable objects by comparison with a catalogue. This paper will mainly focus on the MAJORDOME.
\end{abstract}

Key words: technique: image processory — telescopes GRB

\section{Introduction}

The primary goal of TAROT (Boër et al. 1999) is the detection of optical counterparts of GRB sources. Hence TAROT is an automated ground based observatory able to process both alerts from a variety of sources (CGRO/BATSE, SAX, HETE-II), and normal requests of observations. The TAROT software has 5 different autonomous modules, interacting as shown in Fig. 1.

- The OBSERVATION REQUESTOR is a module that enables anyone to send a proposal of observation to TAROT. The request, will then join the request database.

- The MAJORDOME is the TAROT software module able to optimally schedule observations.

- The CONTROL is the module which is in charge of the actual observatory control and monitoring. Whenever it receives a socket from the MAJORDOME, it actives the CCD CAMERA and images the requested position.

- The CCD CAMERA is for now a commercial Apogee Camera, but we are about to use our own made camera that will be able to read a frame in less than a second.

- The DATA PROCESSING software is composed of TAITAR (Bringer 1997), which is the image processing

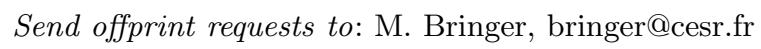

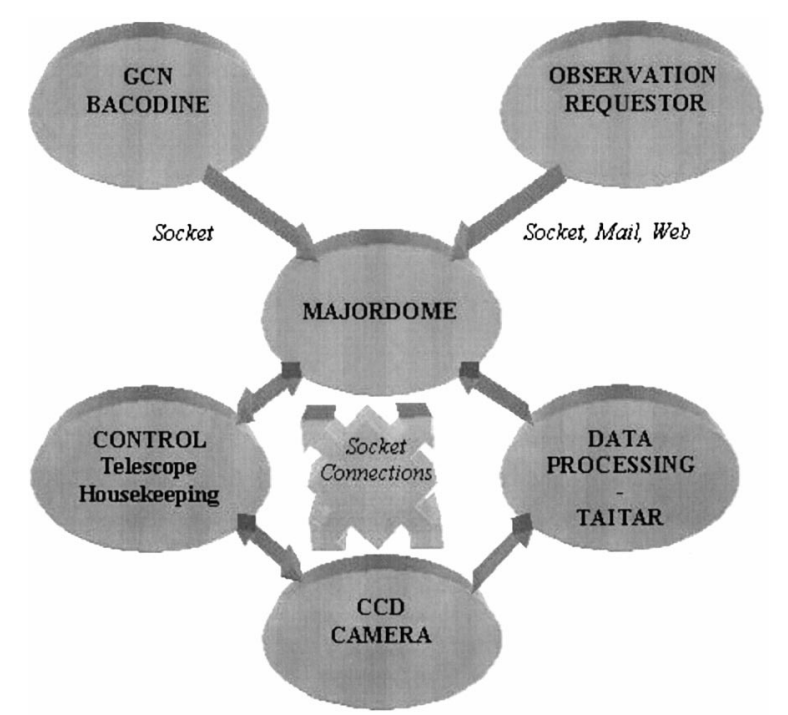

Fig. 1. Connections between TAROT modules

software developed for the purpose of TAROT. The complete analysis of a frame is done in 4 steps (Irwin 1985; Bertin et al. 1996): estimation of the sky background, thresholding, deblending, and photometry. TAITAR then compares each object found on the new frame with the TAROT database in order to detect a variable source or an optical counterpart of a GRB source as soon as possible (i.e. within few minutes). We have also developed a cloud detection algorithm based on flux measurement of reference sources evenly spaced over the sky which allows us to give a map of the cloud coverage, and an automated focus determination.

We present hereafter the MAJORDOME module, and the various observation modes.

\section{The MAJORDOME}

The MAJORDOME is the software that enables TAROT to optimally schedules observations, as well as alert observations. In the routine mode, The MAJORDOME implements several algorithms in order to ensure a maximum efficiency of the observations. Objects should be observed at minimal airmass (unless they have other constraints), and the number of possible observations should be maximized, according to various parameters such as the Moon, user constraints, observations types (periodic, single, etc.) 


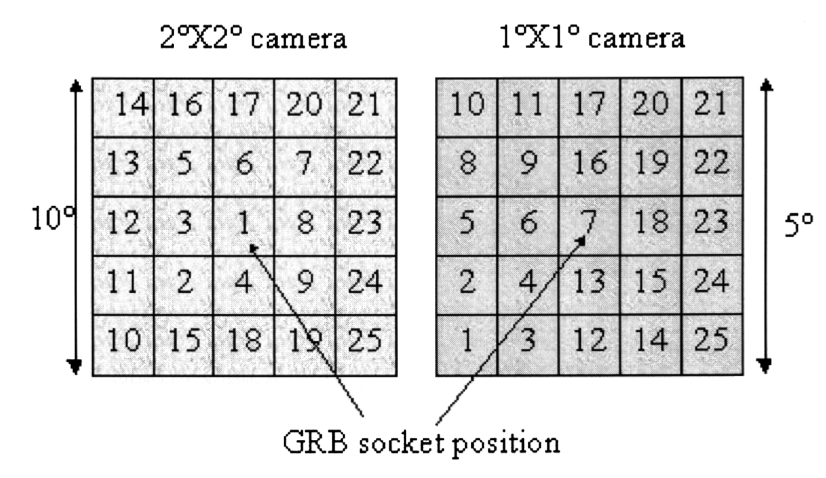

Fig. 2. Mosaic for large error box

and priorities. Whenever an alert occurs, the routine program is interrupted, and its program is activated. A forthcoming paper will focus on the MAJORDOME algorithm.

\subsection{The MAJORDOME in routine mode}

In a routine mode, the scheduling program is divided in 2 parts:

- The selection procedure.

- The run procedure.

The selection procedure selects from the request database all the visible objects for the next night. The requests may be entered at any time (thanks to the OBSERVATION REQUESTOR) and have a lifetime of 1 year. Hence, we have a list of all the possible observations for the next night at our disposal that goes through the run procedure. The core of the run procedure relies on a sequencing algorithm performing pairwise interchanges (Baker 1979) between observation tasks, in order to minimize an evaluation function taking into account parameters such as airmass, proximity of the Moon, etc. The first observations to be scheduled are the strongly constrained requests in reverse priority order. The MAJORDOME then schedules periodic requests and finally the non constrained requests. Their actual date of execution depends on the parameters described above (air mass, moon, etc.). We then have a time table optimized for the night. In case of an interruption due to an alert or bad weather conditions, the run program is reactivated, and a new schedule recomputed for the remaining of the night, taking eventually into account follow up observations if an alert occurs.

\subsection{The MAJORDOME in alert mode}

Whenever a GCN/BACODINE (Barthelemy et al. 1999) alert occurs, the MAJORDOME quits its routine program and instantly starts imaging the GRB position. This takes 3 seconds at most. We have decided to follow the alert types shown in Tab. 1 in descending priority order.

For a large error box as BATSE provides, the MAJORDOME will generate a mosaic of 25 frames covering $25 \mathrm{sq}$ deg for the present camera and $100 \mathrm{sq}$ deg for the next camera available in May 1999. TAROT will image the mosaic until the MAJORDOME receives a higher priority alert.
Table 1. TAROT follow up observations with priorities (1 being the highest priority level) and error boxes radii

\begin{tabular}{|c|c|c|}
\hline ALERT TYPE & PRIORITY & ERROR BOX \\
\hline SAX/WFC & 1 & Few arcmin \\
\hline XTE/PCA & 2 & 1 to 10 arcmin \\
\hline BATSE/LOCBURST & 3 & $1.6^{\circ}$ \\
\hline BATSE/ORIGINAL & 4 & 5 to $10^{\circ}$ \\
\hline ALEXIS & 5 & $\sim 0.5^{\circ}$ \\
\hline
\end{tabular}

We have decided to start our mosaic at the bottom left for our $1^{\circ} \times 1^{\circ}$ field of view camera because it is better to image first the setting objects. For a bigger field of view, it is better to start from the center because, $50 \%$ of the BATSE/LOCBURST GRB sources are found in a $1.6^{\circ}$ radius error box. Today with the commercial camera, TAROT needs 25 minutes to image the whole mosaic. When the next camera will be operational, it will take 5 minutes to produce the mosaic, and only 1.8 minutes to image $50 \%$ of the BATSE alert error box.

\section{Conclusions}

We have developped different softwares modules to achieve the TAROT primary goal which is the detection of GRB optical counterparts and this work has lead to establish new methods to optimmally schedule observations through what we have called the MAJORDOME and an image processing software TAITAR (discussed in a forthcoming paper). The next steps for the MAJORDOME will be to optimally program observations over a larger scale of time as a week or a month. Though, each run procedure will generate a time table optimized for the next month for instance. We also work on the possibility to schedule observations in non-ideal conditions (i.e. during Moon time, between nautical and astronomical sunset). An interesting possibility will be the use of the cloud coverage map to perform observations in selected parts of the sky. The tool will then be of great interest for every automated ground based telescope.

\section{References}

Baker K., 1979, "Introduction to sequencing and scheduling", Duke university, Wiley J. \& Sons (eds.) Inc., ISBN 0-47104555-1

Barthelemy S.D., et al., 1999 (this volume)

Bertin E., Arnouts S., 1996, "Sextractor: software for sources extractions", A\&AS 117, 393

Boër M., et al., 1999 (this volume)

Bringer M., 1997, "Traitement des images d'un telescope grand champ", TAROT-CESR-LOG-RP-002 (in french)

Irwin J., 1985, "Automatic analysis of crowded fields", MNRAS 214, 575 